\title{
Group and individual time management tools: what you get is not what you need
}

\author{
A. E. Blandford \\ School of Computing Science \\ Middlesex University \\ Bounds Green Road \\ London N11 2NQ
}

a.blandford@mdx.ac.uk

\author{
\& T.R.G.Green \\ Computer Based Learning Unit \\ University of Leeds \\ Leeds LS9 2JT
}

\section{Abstract}

Some studies of diaries and scheduling systems have considered how individuals use diaries, with a view to proposing requirements for computerised time management tools. Others have focused on the criteria for success of group scheduling systems. Few have paid attention to how people use a battery of tools as an ensemble. This interview study reports how users exploit paper, personal digital assistants (PDAs) and a group scheduling system for their time management. Like earlier studies, we find many shortcomings of different technologies, but studying the ensemble, rather than individual tools, points towards a different conclusion: rather than aiming towards producing electronic time management tools that replace existing paper based tools, we should be aiming to understand the relative strengths and weaknesses of each technology and towards more seamless integration between tools. In particular, the requirements for scheduling and those for more responsive, fluid time management conflict in ways that demand different kinds of support.

KEYWORDS: diaries, calendars, usability, time management, work context, group scheduling systems

\section{INTRODUCTION}

People use many types of tool to help them manage their time, and they use those tools for many purposes. The range includes the activities traditionally supported by personal diaries and 'to do' lists, and the co-ordination activities that shared diaries are designed to support. Electronic diaries and group scheduling systems have been studied extensively (e.g. [1,2,3]). Almost without exception, these studies have identified limitations of the current generation of tools and proposed enhancements, to the design or the way they are used, that would overcome the limitations. Each 
study has typically focused on one aspect of use - whether that be privacy issues [2] or support for intention formation and prospective remembering [3]. There has been little research on how ITbased tools are combined with traditional tools. Each study of diary use has contributed to a more mature understanding from some perspectives, but has also missed important points.

The study reported here takes a broader perspective, investigating how people use a suite of tools to support their personal and interpersonal time management. These tools include paper, electronic and other media. The principal aim is therefore not to prescribe procedures or tools, but to describe current practice, as reported by respondents, and use that as a starting point for a discussion of the nature of time management tasks and the requirements on tools to support users in this aspect of their work.

The study is based on the results of interviews with sixteen members of staff in a Computer Science department in a British university. While this user population is likely to be more technically aware than a broader cross-section of the community, so that their use of tools is relatively sophisticated, we find that the take-up of technology is lower than might be expected, and respondents each make use of a diverse set of tools.

All respondents, besides their own personal time management tools, had use of a commercially available shared diary system: ON Technologies' Meeting Maker. This tool allows each user to maintain their own diary which can be accessed by all other members of the department. As well as individual diaries, there is a facility to view a group schedule as a set of time strips, each showing what times one individual is free or booked, but not including any details of appointments. This is designed to support group scheduling.

This group of users was selected as a focus for the study because the shared diary system had been in use for an extended period (over two years) by the department, so use could reasonably be expected to have stabilised to a point where novelty was not a factor in people's responses, and users' accounts of how they used it would be fairly mature. The study covers their use of both Meeting Maker and other tools.

The focus of this study is on the use of diaries by individuals, as personal technologies to support their work, not the use of a diary as a repository for organisational memory as discussed, for example, by Kovalainen et al [4]. Inevitably, individual use extends to some collaborative activities, as each individual's work depends on co-ordination with colleagues, but group scheduling is also not the primary concern. However, as one of the themes that emerges from this work is that diary use cannot be understood without reference to the context of use and the use of other tools, both the use of shared diaries and the use of diaries for organisational memory influence the individual's use of their diary.

As one might expect, as a study that focuses on 'description' rather than 'prescription' (describing what actually happens, rather than what should happen), we find many cases of bending the rules, of people finding work-arounds, and of people finding new uses for existing tools.

It should be noted that in this paper we use the term 'diary' to refer to purpose-designed tools (paper and electronic) that allow people to enter information about future events and commitments or as a record of past activities; elsewhere, such tools are often referred to as 'calendars' - a term 
that we use to refer to pre-printed time-based information, or when referring directly to the work of others who use that term. In addition, we use the term 'shared diary' to refer to a diary system that is accessible to others, and 'group scheduling system' for a diary system that supports the specific task of scheduling meetings by co-ordinating information from multiple diaries. Scheduling is one task supported by diaries - that of allocating a particular time period to a particular activity, for one or more people.

\section{BACKGROUND}

Time management tools have been studied extensively over many years. While early studies focused on single user systems, more recent ones have considered shared diary systems and group scheduling activities. Time and time management have fascinated many authors; for example, Jackson [5] uses the lack of a simple mapping between weeks, months and years as an example of structural mismatch in software specification. From another direction, Kullberg [6] and Mueller [7] use particular requirements for diaries as a focus for developing novel technical solutions, regardless of whether or not the resulting artefacts are in practice usable. Here, we are concerned primarily with user behaviour and requirements. In particular, we have the opportunity to establish users' perceptions of the impact of using a shared diary on their individual behaviour.

\subsection{Identifying requirements on time management tools: focusing on users}

The earliest studies of diary usage targeted at computerisation are probably those of Kelley \& Chapanis [8] and Kincaid, Dupont \& Kaye [1]. Despite finding great diversity in the time management behaviour of the individuals in their studies, both sets of authors were optimistic about the great potential of computerisation. Kincaid et al [1] present a substantial list of recommendations for electronic diary systems, for example that:

- There should be daily, weekly and monthly formats for display.

- There should be no restriction on the amount of text per entry.

- The range of the diary should be unlimited, etc.

One interesting point about these recommendations is that the electronic diary system that was available to all users in the study reported here (Meeting Maker) satisfies all the requirements proposed by Kincaid et al bar one. The only requirement not satisfied by Meeting Maker is that the "format should be as unstructured as possible, requiring only that optional begin/end times be in some fixed location". Kincaid et al express the view that "Automatic schedulers offer the opportunity to provide extremely powerful and useful functionality in an electronic system, but most of the currently available models have serious shortcomings that limit their usefulness [...] It is the authors' hope that this paper will be of value in correcting this situation". The study reported here indicates that their hope has not been entirely fulfilled, probes some of the reasons for this, identifies some further requirements on electronic diary systems but also concludes that there is no one tool to serve all purposes, and that the aim should be improved integration across tools.

Beard, Palanlappap, Humm et al [9] build directly on the work of Kincaid et al in their design of a visual diary, VS, that serves also as a group scheduling system. The system they describe shares 
most features in common with Meeting Maker, including the visual structure and the mechanism for scheduling meetings. The most important exception to this rule is that whereas Meeting Maker presents a composite schedule (showing multiple diaries) as a series of time strips, VS shows them all overlaid, using transparency to indicate importance (more opaque if more important). Using their scheme, the best times for appointments are the most transparent when the diaries of all participants are overlaid on each other. A related point is that, whereas Meeting Maker allows the user to code events using a personally defined colour scheme, VS requires users to attach a priority to events, and sets the transparency of the label accordingly. Beard et al evaluated VS using both a traditional experimental paradigm, comparing the use of VS for scheduling against traditional manual scheduling in terms of time taken and error rates, and a longitudinal field study. The field study took place over four weeks and involved fifteen participants. The fifteen subjects used were the subset of the people who had access to the system who actually made use of it for scheduling during the four week period. Results of this study were largely positive. Of particular relevance to the current study are the following points:

- The term 'priority' might denote how important or how easy to move an appointment is; this ambiguity caused confusion. Importance and movability are just two of the properties of appointments that respondents in the current study reported using when adjusting their schedule in response to new information or demands.

- Users were aware of the impression of themselves that they were projecting through their diaries, and would sometimes label an event with a higher priority than it actually merited in case the people they were scheduled to meet looked at their diaries and formed the "wrong impression'. Similarly, in the current study, users reported a range of strategies for projecting 'acceptable' images of themselves through a shared diary.

- Users all wanted VS to be integrated with their desktop environments, to support data sharing. Integration was highlighted as an important requirement by many respondents in the current study.

A more theoretically driven study was conducted by Payne [3], who focused attention on the tasks of prospective remembering (i.e. remembering to do things in the future) and of managing interdependent intentions - for example, that organising a meeting involves inviting participants, booking a room, booking refreshments, etc., and that if the meeting then gets cancelled, various other actions have to be taken, or not taken, as a consequence. Payne interviewed 20 professional scientists, all working within the same organisation. In his semi-structured interviews he asked set questions that were open-ended enough to encourage interviewees to talk freely about their time management behaviour. The results were a starting point for conducting what he termed a 'Task Structure Analysis'. The central premise of this was that the primary role of diaries and to-do lists is to support prospective remembering. He then considered ways in which the use of any external memory aid affects that task - for instance, by influencing the formation of intentions or supporting scheduling. Taking the intention as the primary conceptual entity in the task of prospective remembering, Payne then conducted a Task Entity Analysis, considering the nested structuring of intentions, dependencies between intentions and temporal constraints on intentions.

Payne's approach could be summarised as eliciting the user's model of time management through his Task Entity Analysis and seeing to what extent it was matched by the device's model of time management. The extent to which different diaries do or do not represent these entities was used as 
a basis for discussing design requirements on diary systems. In common with other studies, Payne makes design suggestions that appear to be based on the assumption that total computerisation is desirable and achievable.

\subsection{Identifying conditions for success of groupware: focusing on organisations}

Focusing on group use, Grudin and Palen [10] and Brown and Crawshaw [11] report investigations of electronic diary systems. Both investigations concerned the acceptance or rejection of shared systems, and in both cases the authors seem to be evangelising the possibilities of such systems. Grudin \& Palen [10] identify success factors as including perceived usability and usefulness to individuals, peer pressure (to facilitate group scheduling) and the need for a technical infrastructure that supports activities adequately. This work is followed up by Palen [12,2], who goes on to identify requirements for effective groupware design. Brown and Crawshaw [11] focus more on selecting between the commercially available products and the importance of user training. Consequently, their considerations are biased to the electronic systems, and although their conclusions concern users' attitudes and purport to represent the social and technological influences on those attitudes, they fail to consider either the relationship between the user's model and the device's model, or the social and technological advantages of other time-management resources such as paper diaries. These advantages are considerable, so that for a clear picture of the complex pattern of choices and activities it is essential to study the needs of users within the organisational context, not a single type of device.

\subsection{Summary}

To summarise past studies on time management tools: some typical task needs have been identified, and mismatches between the user's model and the device's model has been noted, but research has mainly concentrated on one tool at a time. Relatively little work has considered how individuals select a particular tool from a battery of available tools, and how they integrate the use of different tools within their overall daily activities. In this paper, we report on an interview study of time management tools, considering in particular how tools are used together, and how users manage their tool use.

\section{METHOD}

This study is based on the results of interviews with 16 respondents. All are members of staff in a Computer Science department in a British university. The university is housed on several widelyseparated campuses; while some staff (typically administrators and lecturers) spend most of their time at their base campus, managers are expected to travel extensively to attend meetings at other campuses. In addition, both lecturing staff and managers travel fairly frequently - e.g. to conferences, or to maintain collaborative links with overseas universities.

All staff have use of Meeting Maker (see figure 1 for an example of a weekly screen display from this system). This tool allows each user to maintain their own diary which can be read, but usually not written to, by all other members of the School through the use of a "proxy" feature. The default set-up is that all other school members are read-only proxies. In principle, managers can modify 
their settings so that their personal assistants (PAs) have write access to their diaries too; in practice, most PAs have evolved the practice of logging in as their managers, as they find this easier and more reliable.

Figure 1 here

FIGURE 1: Example Meeting Maker screen (weekly view)

Staff were approached to participate in this study with the aims of achieving a reasonable representation of both male and female, a broad age range and a cross-section of job-functions. There were nine male and seven female respondents; their roles were:

- 4 managers

- 4 personal assistants (PAs)

- 3 group leaders / lecturers (i.e. lecturers who also have a substantial administrative role, and therefore the support of a PA)

- 5 lecturers and research fellows

Our interview study is modelled on Payne's [3] study, using many of the same questions. Our subsequent analysis, however, focuses further on properties of tools and the broad range of time management tasks people are using these tools for. A copy of the interview form is included as an appendix to this paper. Most interviews lasted between 30 minutes and an hour.

\section{RESULTS}

The results are summarised under 4 headings:

1. The various types of time management tools employed, including very informal tools such as human memory, and paper-based and computerised tools.

2. What people use time management tools for, including, but not limited to, time management.

3. The issues that arise from the use of tools - considering individual use and the impact of group access on individual use.

4. A discussion of the implications of this work for the design of time management systems.

\subsection{Technologies and information sources}

We start by describing the technologies used by respondents to support their time management. Results are summarised in table 1. We have chosen to describe them under four column headings in order of increasing structure to the information being represented. 


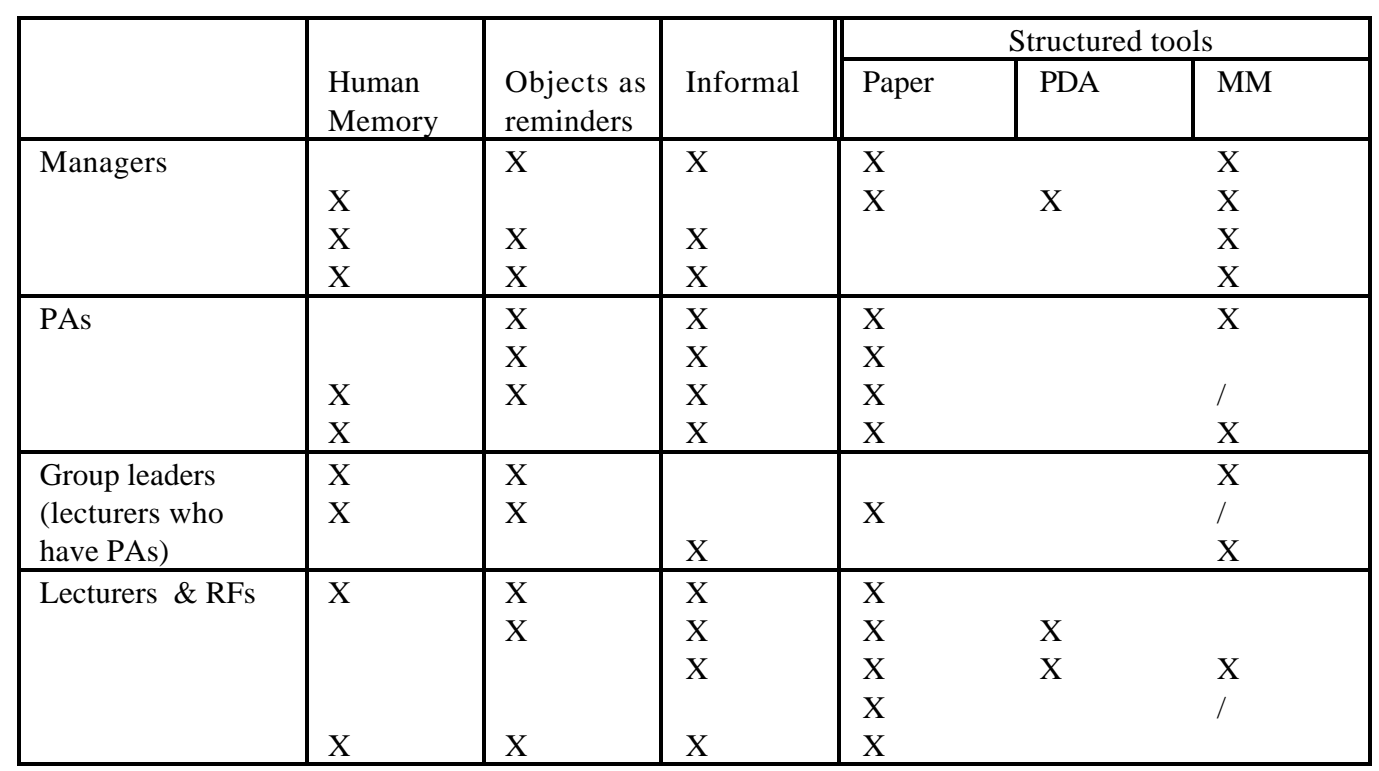

Table 1: technology types used by informants

$X$ indicates at least one instance in our sample; / indicates that the individual reports entering information in Meeting Maker so that others can access it, but not using Meeting Maker for their own time management.

\section{Human memory}

Several of our informants reported using their memories as an important tool. Lecture schedules, being repetitive, are often remembered by lecturers; even for non-repetitive events some informants claim to make little use of any external aide mémoire and to be able to remember most of their appointments.

In addition, some informants are aware that they rely on their colleagues' or their spouse's memories, asking to be reminded or simply being given an unsolicited reminder as an appointment becomes imminent.

As Payne [3] emphasises, with his focus on prospective remembering, it is essential that people remember intentions at the point when they become current; one thing that became clear in the current study is that many people rely on their memories for future events too, and use that memory in intention formation and scheduling.

\section{Using things as their own reminders}

Several informants mentioned using the physical objects themselves - i.e. the work materials as reminders of things to be done. One leaves 'piles of papers around the house' so that he trips over them; others use strategically placed piles in the office as well as files and folders as reminders, typically by putting them into a briefcase or in a pending file. One informant explicitly mentioned using the queue of people outside his door as an appointments reminder. While not a time management tool in the usual sense, this is part of his standard way of working. 


\section{Informal reminders - mobile and fixed}

Very nearly all informants use other informal methods to remind themselves. Some are ad hoc; others are routinised.

Some informants make reminders and to-do lists on the backs of their hands; one carries a pen at all times for that precise purpose. Others make notes on scraps of paper. A few informants use unstructured word-processor files to maintain to-do lists.

Many informants also use post-its, which are pieces of paper with a fixed location. Sometimes post-its are used in a purely ad hoc way, like the scraps of paper just mentioned, but their fixed location allows them also to be used in more formal ways: they can be attached to screens, doors, or desks in standardised positions - for example, to indicate priority of the item on the note. One informant maintains a two-dimensional to-do list from post-its on the desk, with the most urgent (axis 1) and important (axis 2) items nearest the computer keyboard (and the bin strategically placed near the furthest corner of the matrix).

One informant uses a mobile phone as a to-do list, by entering all the numbers to be telephoned each day into its memory. Some informants use the answerphone as a time-management tool, by leaving messages for each other or as reminders for themselves. A similar use is made of electronic mail. Most respondents make heavy use of email to request or confirm meetings, send reminders, etc. These examples of technology use exemplify the co-adaptive nature [13] of people's adoption of technologies: that tools that were originally designed for one purpose get adapted and used in ways that their designers would not have anticipated.

\section{Methodical reminders - paper and electronic}

Low technology, but structured, reminder methods are in common use. These include calendars on the wall, wall charts such as year planners, and methodically-used paper pads or booklets. The purpose-made paper-based diary is the most common of these tools. These methods are usually part of the personal or office routine, and are often consulted at set intervals.

Certain information, such as the University diary and timetables, is circulated in printed form, or sometimes email, and retained by staff for reference.

Three respondents reported using personal digital assistants, or PDAs. All three use Palm Pilots, which include a diary, a to-do management tool and a database of contacts.

Most respondents in the study make use of Meeting Maker to some degree: as their principal diary; as a summary of the main events from their diary (for public information); or on an ad hoc basis.

\section{Summary}

The different types of technology respondents report using are summarised in table 1. As this table shows, all respondents use at least one structured diary, whether electronic or paper and many (39\%) use both. Most respondents (75\%) still use paper diaries - whether at work or just for home use. About half ( $56 \%$, indicated by ' $X$ ' in the MM column) use Meeting Maker as a primary record of events, while a further 3 people (19\%, indicated by ' $/$ ') record information into Meeting Maker to make it accessible to others. Significantly, almost all respondents also rely on other unstructured 
tools to support their time management; these include both external artefacts and their own memories.

The total usage of electronic tools reported here is higher than that found by Jones \& Thomas [14], who present the results of a pilot study of 'personal information management tools' that concludes that few (less than $20 \%$ of the 23 subject in their informal survey) make use of any electronic information management aids, but that most people (more than 50\%) use two or more aids of some sort, of which the to-do list is the most common. This difference can be accounted for by the small size of both samples, the relative technological sophistication of the users in the current study, and the free availability of Meeting Maker to all users in this study. The current study provides evidence to account for the finding of Jones \& Thomas that take-up of electronic tools is lower than earlier researchers predicted.

The various types of tools contrast on several important dimensions:

- People's own memories are immediately accessible to them, for both reference and update, but are prone to failure (forgetting) and are inaccessible to others.

- Things that serve as their own reminders are typically accessible at the time that they are needed (though not reliably so), demand little maintenance and may be inspectable by others.

- Informal tools are typically accessible, but require explicit maintenance. Some are inspectable by others, but many are not.

- Structured tools are often the least accessible, due to fixed locations, and demand explicit maintenance. Shared tools (both paper and electronic) can be accessed by others.

These dimensions - of how portable, accessible, shareable and updateable tools are - are some of the important factors identified by users in this study as determining their choices of technologies.

\subsection{Uses of time management systems}

In our summary of tools used, we showed that most users adopt general-purpose tools and tools that were designed for other purposes (bits of paper, mobile telephones, etc.) to support their time management tasks - particularly prospective remembering. In this section, we consider the issue from the other side: we outline both the time management activities that respondents discussed, and also the other uses to which they put the tools that are designed primarily to support time management.

\section{Reminders of appointments and to-dos}

The first, and probably most obvious, use of tools is to support prospective remembering: that is, appointments and to-dos. We use the term "appointment" to refer to a meeting or event that has a designated start time and expected participants, and sometimes a location and various other properties. A structured diary of some form is kept by all 16 respondents to record appointments. Some maintain separate diaries for home and work use; people adopt different strategies, as discussed below, regarding what appointments get noted where. 
Many activities (e.g. preparing a lecture, 'phoning someone, working on a report) have no particular time-slot allocated to them. These are generally managed through some form of to-do list or other non-time-based technology. To-dos gain a time-dimension when there is some deadline that has to be worked to.

\section{Other time-based information}

Many respondents reported recording various kinds of information other than appointments in their diaries. These include:

- A note of which week of the semester it is, coursework deadlines, examination dates, and similar information.

- A note of when particular colleagues are away (for example, if the person is deputising for the individual who is away, or if the two people normally work closely together).

- Pointers from the diary entry to supporting materials (e.g. papers for a meeting, an electronic copy of the agenda or a note of the contact details for the person being met).

- Reminders of deadlines and of other actions to be performed on the day.

- A note of events, such as people visiting the department that the individual might want to meet briefly but does not have an appointment with.

- Occasionally, to-dos get noted in diaries.

- One respondent notes birthdays of close colleagues in his diary.

What we see here is a large miscellany of contextual information that users evidently feel the need to record. Previous studies have hardly mentioned any such diversity. Time management does not consist solely of formal appointments and simple reminders. Not surprisingly, users are restricted by the current generation of interactive systems.

\section{Group uses}

As well as individual use, people make use of the shared facilities of the Meeting Maker diary. For shared diaries, we can identify five classes of reported use:

- Many respondents report using shared diaries to help in identifying a suitable time for a meeting.

- PAs who have a 'minding' role need to know where their manager is expected to be each day, so that they can remind them and provide any necessary paperwork; also, they need to be able to make new appointments on behalf of their managers;

- Health and safety regulations require that the locations of individuals should be known in case of emergency; 
- Some users use the shared diary as an information resource - e.g. looking at someone else's diary to get the details of a meeting the information-seeker is due to attend without having to ask about it;

- Several people also reported curiosity-driven reference to others' diaries. For example if they haven't seen a particular colleague for a while and wonder where they are, they may consult their diary.

The first two of these uses are ones for which the product was designed. The third is an additional one for which it was installed (i.e., managers perceived this as being a beneficial side-effect of the use of a shared diary system); the remaining two reported uses are examples of co-adaptive behaviour: that people discover uses for the technologies that were not originally intended but that become part of people's normal working practices. The group scheduling system is serving as a repository for organisational memory. This information provides an additional resource to the individual in managing their own time, and satisfying their own curiosity, but also places additional demands on the individual, in term of making information about their activities publicly viewable.

\section{A record of past activities}

Several people reported using diaries and pads to keep a record of past events. Uses include the following:

- Simply keeping an account of the way time has been spent, to allow the user to reflect on their own time management and account for their use of time to more senior managers.

- For calculating mileage claims. This was reported by most of the staff who regularly travel to other sites.

- To support future planning, on the grounds that there are various things that happen annually, so a record of the past is a reasonable predictor for the future in certain respects.

- For recording the times and outcomes of meetings, for reference in case of queries.

- Out of work, one respondent reported keeping a diary of activities related to a particular hobby, but this was the only mention in this survey of using a diary as a record for non-work-related information.

Most of the reported record keeping makes use of the same tools as forward scheduling; while it is not a time management activity per se, it exploits the fact that appointments recorded as future events typically become past events, so that a fuller record can be maintained just by annotating diary entries.

\subsection{Use and usability issues}

Having listed the technologies used for time-management and described the uses of these systems, we now consider issues raised about the ways the technologies support the actual uses. 


\section{Individual use}

Individuals use a broad range of technologies to support their time management. In describing their use of particular technologies for certain purposes, respondents made reference to important global properties of those technologies, as well as more specific features such as how expressive they are or how easy it is to integrate information across tools.

The global properties of technologies are the features that most influence their usefulness in context: in particular, portability, accessibility and the ability to locate technologies in prominent places are important. In addition, various properties of technologies in use, such as the visual structure of the display or how much space they allow for a diary entry, affect their overall usability.

\section{Portability}

Most of the managers and PAs cite accessibility of a shared diary from different places (particularly when travelling abroad) as the greatest benefit of using Meeting Maker. These people generally travel with a portable computer and the means to make a connection to the Meeting Maker server from almost anywhere. The strengths of the shared diary are that it can be accessed by different people and from multiple locations.

Conversely, for staff who travel little, or who travel without the facility to log in remotely, the relatively fixed location of their Meeting Maker diary is generally seen as a disadvantage. Most people access it only from their desks, though a few also report accessing it from their home computers; they have adopted a variety of strategies for dealing with situations where they are asked to make appointments while they are away from their desks (e.g. agreeing a date for the next meeting while in the current meeting, or agreeing to see someone if stopped in the corridor). For example:

- some users methodically take a printout of their current diary (printing out the next 2-3 months) whenever they attend meetings, so that new entries can be added manually then transcribed to Meeting Maker later;

- some users ask people to make an appointment through their PA or an administrator;

- some either make a note on the back of their hand (often literally) or aim to remember the conversation until they get back to their office and can confirm the appointment time;

- some operate fixed office hours and ask people to come and see them at that time, or maybe create a large time window in which they might be available to visitors, without guaranteeing to be available throughout that window (one respondent calls this practice "stealth time management").

As shown in Table 1, a small proportion of users use a PDA in conjunction with Meeting Maker, transferring information between the two regularly, to combine the portability of the one with the shareability of the other. 


\section{Ready accessibility}

As well as the issue of portability, several users raised the issue of rapid access. If the computer is not on, or if Meeting Maker is not running, it can take anything from 15 seconds to 2 minutes to access Meeting Maker in order to either read existing entries or make new ones. This contrasts unfavourably with both PDAs and most paper- and head-based systems. If the user is away from their computer, a desktop based system such as Meeting Maker becomes even less ready-to-hand.

\section{Visual salience in the work setting}

The importance of location cannot be underestimated. Many respondents talked about the way they place items relative to their main work area, with paper diaries left open in front of them on the desk, post-it notes placed near their computer screens and important papers left in a visually prominent position. A reason cited by several users for not running Meeting Maker routinely is that the Meeting Maker window clutters up their screen: either it is at the front, obscuring the items currently being worked on, or it gets obscured by other windows and hence is difficult to access quickly.

While visual salience might be associated with portability (a portable item can be placed at any chosen location), it is a separate issue that also needs to be recognised as being important.

\section{Fluidity of the visual structure}

Electronic organisers (including both Meeting Maker and the Palm Pilot diary used by respondents in this study) typically have a more fluid visual structure than a paper diary; for example, on the Palm Pilot the time window being displayed can vary dynamically as new diary entries are made, and on most systems (including Meeting Maker) the display can be scrolled in a fairly unrestricted way, so there are no fixed reference points. Several people reported entering appointments in the wrong day, week or year using electronic diaries. Only one reported making the same error when using a paper diary. They generally accounted for this by saying that there were fewer cues to location in an electronic diary (e.g. Monday is not always at the top left of the page).

Some respondents commented on the size of the displayed area for an activity; while one respondent considers it an advantage that an extensive piece of text can be used to describe an activity in a small time slot (though most of that text will not be immediately visible on the screen without "opening" the entry), most consider it a disadvantage that the text is hidden ("You can't write smaller to fit more in").

\section{Local vs. global view}

Some respondents report keeping a larger time window visible (e.g. an monthly view open on Meeting Maker, or using a month-to-a-view paper diary) so that they can maintain better lookahead (e.g. to plan for meetings, or buy food for dinner parties, or simply not get overwhelmed by commitments). One user of a PDA commented that it was difficult to see the 'big picture', and one user of a paper diary said that he had occasionally forgotten Monday morning appointments because he had not turned the page in his diary soon enough. The need for a detailed local view supplemented by a more general global view (of the future, though not of the past) is discussed by 
Payne [3], who postulates that a fish-eye view might be a good solution. Some tools offer lookahead naturally, while others do not support it at all.

\section{Scarring}

Paper-based tools keep a record of changes through "scars": obvious crossings out and corrections. Many users of electronic technologies value their lack of scarring ("It keeps tidier"), but a few explicitly implement scarring by annotating diary entries (e.g. "CX") to show that they have been cancelled or moved. One particular comment, referring to Meeting Maker, is that it does not provide an "undo" facility, so if an event is accidentally moved or deleted it can be impossible to recover from the error locally. While viscosity [14] is normally considered undesirable, this is an example of a case where low viscosity can provoke errors that are hard to recover from. People have developed sophisticated strategies for such error recovery: for example, guessing who else will be at the meeting in question and looking at their Meeting Maker to find out the meeting details.

\section{Prioritising intentions}

For to-do lists the most important property discussed is how easily to-dos (or intentions) can be prioritised. Most people use their heads for this, or simply annotate a paper list (e.g. writing or underlining items in red, or doodling asterisks next to urgent items). The facility provided by the Palm Pilot of allocating a priority level of 1-5 is not generally valued because what matters is not how many urgent and important tasks one has, but which is the most urgent and should be dealt with first.

\section{Expressiveness of technologies}

People record information of various types in their diaries, including:

- scheduled meetings with start times, end times, locations, etc.

- blocks of time for doing solo activities

- "pulse" events (of the "must get that done this afternoon" variety)

- notes of deadlines

- notes of other happenings that might impact on the diary user (e.g. meetings that they should be aware of, visitors to the School, when close colleagues are away)

Most computerised technologies force entries to conform to types that do not match the user's types. For example, Meeting Maker only distinguishes between "meetings" and "activities", and this distinction is not a user-meaningful one (it refers, not to any semantic difference, but simply to whether or not a meeting was set up via Meeting Maker). In general, there is no distinction between scheduled meetings, more flexible time-based intentions and other time-based information, and users of Meeting Maker are forced to find work-arounds that mean the system not used in the way originally intended by its designers. 
Some of these work-arounds are disarmingly inventive. For example, some users create information notes - a facility not supported by Meeting Maker - by disguising them as real events, entered at times when no real event would be booked, such as very early in the morning (Figure 1 contains two examples: "Anne away" and "German").

Events have attributes of "importance", "definiteness" and "movability" (as noted by Beard et al [9]). Users of paper diaries often mark some of these properties by their choice of writing style, pen colours, or annotations (e.g. underlining or marking with an asterisk). Much of the information also remains implicit (e.g. users will not even consider whether or not an event is movable unless the need arises). This implicitness can cause difficulties - for example, when someone is trying to find a meeting time to suit several people, or if a PA is expected to know where their manager is but the diary has a double booking (which is the manager actually at?).

\section{Explicit and implicit information}

Electronic diaries enforce explicitness. They commonly insist that the end time or duration of a meeting shall be specified as well as the start time. Many people view that as a difficulty. For example:

- On the PDA used by one of the respondents, meetings have a default length of one hour. The respondent reported critical incidents where he had entered a meeting that was actually expected to last 2-3 hours, but had forgotten to change the duration attribute, and had then accidentally scheduled another meeting to follow it (i.e. only allowing an hour for the first meeting).

- In practice, people often do not commit to a particular length of meeting in advance, but either let the meeting "run its course" or allow the requirements of a following meeting to define the length of this one. Conversely, paper diaries often contain entries such as "Do activity B after activity A" - i.e. B has no particular start time, but will be done whenever A has finished. Current electronic diaries do not allow users to express these kinds of ideas, leaving a certain ambiguity: for instance does the entry "A 9.30-10.30 ; B 10.30-11" mean B immediately follows A, regardless of how long A takes, or does it mean B starts at 10.30am?

This difficulty is recognised by Cooper [15], who argues that there are two types of time-based information: deadlines and ongoing processes, and that the appointments that are implemented in all existing diary systems are inappropriate for both of these categories. As discussed, real appointments have start times but rarely have pre-determined end times. In addition, real appointments may notionally overlap, but many diary programs do not permit this; neither do they allow an appointment to have a start time and a preparation time (e.g. travelling time to get there). No respondents in the current study reported difficulties regarding overlap, because this is not a restriction imposed by the tools they use; occasional mention was made of the travelling time problem.

Existing electronic systems are well suited to one particular task, namely explicit scheduling. While most users find this explicitness a hindrance, a few report it as an advantage: that having to make this information explicit helps them to manage their time more effectively. Reference to Figure 2 illustrates the scheduling task, and the support that the display provides for this task. The visual structure clearly indicates that week (a) is going to be busy since it is split into a large number of 
labelled chunks with no gaps between them (except 8-9am on Wednesday and Thursday), that week (b) involves the diary owner in a single extended activity since each day is filled with a single labelled chunk, and that week (c) is relatively free since there are few entries. All three displays show clearly which times are available for booking.

Figure $2(\mathrm{a}, \mathrm{b} \& \mathrm{c})$ here
(a) a busy week
(b) a week away
(c) an uncluttered week

Figure 2: scheduling systems express 'busyness' clearly

In practice, individuals manage their time in much more fluid ways than this 'scheduling' model acknowledges. Time management involves a complex interplay between deliberative scheduling, reacting to immediate, short-term demands and completing substantial tasks that are solo activities. No existing tools support all of these demands in an integrated, coherent way.

\section{Event series}

In the organisation, many events form series - for example, a lecture course, a seminar series, research, teaching and management group meetings. Meeting Maker has a frequency feature that allows the user to set up a series of events at regular intervals, as proposed by Kincaid et al [1], and similar to that implemented in VS [9]. In principle, users should therefore be able to enter information about event series much more efficiently using Meeting Maker than using paper. Indeed, this is one of the advantages of electronic tools highlighted by Palen [2]. In practice, however, few users reported using the feature. Reasons given include the following:

- If each event in the series needs an annotation (e.g. topic of lecture, or room number for meeting) then each needs to be dealt with as a separate event;

- Within a lecture course, there may be a large number of events every week (e.g. two lectures, several lab classes, several seminars); each event in a week needs to be entered separately. Particularly for lecture courses, many lecturers view this as an unnecessary chore; they simply carry the paper copy of their lecture timetable around with them for the first few weeks until it has been memorised, and then work just from memory;

- If events are not at precise intervals that can be expressed as, for example, "the 15th of the month" or "the third Tuesday of the month" then they cannot be entered as a series in Meeting Maker;

- Even if the events do have this property (of being regular in this way), many users are not aware of how to use it; for example the " 15 th of the month" is counted as a monthly event, whereas the "third Tuesday of the month" is counted as a weekly event. Some users believe that Meeting Maker does not support this kind of frequency, because they have failed to locate it.

While the last of these indicates a need - for a more discoverable design or more extensive training - that could be addressed relatively easily, the other user requirements suggest that a different implementation of 'event series' that is less dependent on the notion of 'frequency' would be more appropriate. 


\section{Cachet}

The image of themselves projected by their diaries was mentioned by three users. One user of a PDA said that she felt it helped to project an image of herself as a competent, efficient professional. Conversely, one user of only paper diaries proudly showed his rather battered diary that was covered with small handwriting and doodles, and projected a rather Bohemian image, while another emphasised the importance to him of having a diary that included beautiful colour photographs of wildlife on each left-hand page.

\section{Meeting arrangement}

The main reason reported by respondents for consulting any one else's diary was to facilitate meeting set-up. Indeed, as the name implies, Meeting Maker is tailored towards the setting up of meetings. However, no users of Meeting Maker report using the "meetings" feature.

Apparently the meeting set-up feature was perceived as being unreliable. Obviously, a glitch could waste several persons' time and expose the unfortunate user to hostility or derision.

More significantly, many users have developed the strategy of blocking out time for individual activities, or just so that they can control what meetings get booked; for these users, it needs a human eye and human judgement to assess what time is actually bookable for a meeting of a particular type and what time is not. Therefore, in practice, people report opening others' diaries (as read-only proxies) and finding a suitable time manually, then using telephone or email to set up the meeting. This raises various issues, as follows.

\section{Reliability of diary entries}

A few users reported critical incidents where someone had checked their diary, noted a time with no entry, and booked a meeting with them at that time without independently checking that they were actually free. Sometimes this results in minor violation of social protocols, with the need to repair a misunderstanding. There have, however, been cases where meetings involving externals have been arranged on the basis of the information in a shared diary; this has led to much greater interpersonal conflicts and the need to re-arrange meetings that had appeared to be fixed. The most common case of this has been meetings arranged by administrative staff (PAs and group secretaries) involving academic staff.

Some of the administrative staff report developing a set of beliefs about individuals whose diaries they trust and those who they don't. In one case, this is a "category" belief: "The more senior people are better at keeping their diaries up to date"; in others, beliefs are based on experience of working with particular individuals. In general, as well as learning to maintain one's own diary effectively for group use, respondents have found that there is a need to learn to "read" and use the information in other people's diaries appropriately — both in terms of knowing how much trust to place in information and also knowing how the person organises their life. 


\section{Making intentions explicit in diary entries}

Some users also reported cases where they were planning to prepare a lecture or work on some other "solo" activity that they had not made explicit as a diary entry, and the proposal of a meeting caused a social conflict: that they had set aside a contiguous chunk of time which they did not particularly want to break, and yet to refuse a meeting at that time (when they are apparently "free") would have appeared impolite.

Many users have adapted their diary management practice to block out large chunks of time for solo activities, releasing that time selectively in response to demand. One admitted to having created fictitious students with whom he had frequent meetings marked in his diary. This makes it impossible for meeting times to be automatically identified by the Meeting Maker system. It also forces users to be explicit about the timing of activities that they have always been able to leave implicit in the past. There is a tension between the role of the diary as a public information system and its role as a personal memory aid.

\section{Exploiting the technology for other needs}

Two of the managers interviewed talked about the Health and Safety role that Meeting Maker should play, replacing, as it does, weekly whereabouts sheets. Of the other staff interviewed, only two mentioned anything that indicated an explicit awareness of this requirement. From a Health and Safety perspective, the main requirement is knowing where someone is at the moment, in case of emergency. Inspection of Meeting Maker entries will show that this information is often not made explicit, though in many cases it can be inferred from entries.

The few reports of curiosity-driven consultation of Meeting Maker diaries all appear to be benign (i.e. having no inappropriate motive). However, there are divergent views on what information about oneself should be made public:

- at one end, some users expressed the view that they have no secrets and that it is their duty to make their diaries inspectable by other staff within the school or by higher management.

- at the other end of the spectrum, some users express deep concern about how others, particularly higher management, might use information from their diaries (e.g. requiring them to justify their use of time).

- many users only put entries in their shared diaries that are explicitly work related, avoiding, for example, entering notes about dental or doctor's appointments, even though they consider them legitimate activities to have during the working day. Some put such things in their diaries, but use a personal code so that no-one else would recognise the meaning of the entry. A few users use the "Private" feature provided by Meeting Maker for such entries.

Most users are explicitly aware of the shared nature of Meeting Maker information; some consciously adapt the entries they make for the possible audience, using codes or, conversely, avoiding cryptic notes so that their entries are comprehensible to others; some even write instructions to potential readers of their diaries; for instance, one user's diary contains the entries: "work at home $[\ldots]$ phone me if you need me" and "[...] - who put this note here (was it me?) and 
who is it and what do they want". However, there is a fundamental mismatch between the individual's needs for a diary that helps them remember about appointments and manage their own time and one that provides semi-public information about their activities.

The issue of trusting unknown readers of one's diary, when set against the health and safety requirement, illustrates another mismatch in the purpose of diary entries (e.g. from a health and safety perspective it is important to know when someone is off-site, but the individual might regard this as "private" information). This issue - of trust and privacy - is a focus of the work reported by Palen [2].

\section{Co-ordinating information resources}

British academic life permits no definite separation between home life and work: some teaching takes place in the evenings, people often prepare lectures or do reading at home, there may be evening entertainment of visiting academics, a dental appointment may occur during the "working day", or people may be away at a conference for a few days. Interviewees reported various ways of managing work and home:

- Some maintain just one diary to cover all aspects of life;

- Some maintain a personal diary that covers all aspects of life and transcribe selected items to their Meeting Maker diary;

- Some rely on their memories for home-based information, using a diary only for work;

- Some maintain separate diaries for home and work; the home diary may be shared with other family members.

Few reported having a methodical scheme for integrating information across their various time management tools: integration between to-do lists and diaries is typically done in the head, while co-ordination of multiple diaries is often done in a "crisis management" style: of doing a reconciliation when a breakdown has been detected.

Payne [3] proposes that the acts of reconciling information sources and of inserting new entries provide a mechanism for reminding of up-coming events, and hence serve as an important prospective memory aid. The evidence from the present study is that most people do not place much value on being reminded by this means, and perceive reconciliation as a tedious task, to be avoided wherever possible. One of the challenges for the future is to streamline the co-ordination task for multi-diary users. 


\begin{tabular}{|c|c|}
\hline Requirement identified & Possible approach to implementation \\
\hline $\begin{array}{l}\text { Support memory for future events to facilitate } \\
\text { planning }\end{array}$ & Provide look-ahead \\
\hline Link diary information to other resources & $\begin{array}{l}\text { Provide facility to create pointers of references to related } \\
\text { resources (both electronic and other) }\end{array}$ \\
\hline Provide an augmented record of past activities & Allow addition of information after the event \\
\hline \multirow[t]{3}{*}{ Minimise likelihood of incorrect diary entries } & $\begin{array}{l}\text { Do not permit new diary entries in the past without some } \\
\text { warning }\end{array}$ \\
\hline & Have fixed points on display to help user orientation \\
\hline & Implement indication of how far in the future a new entry is \\
\hline Display appropriate detail & $\begin{array}{l}\text { Implement a means of displaying detail about event without } \\
\text { either opening it or adjusting display area (e.g. roll-overs) }\end{array}$ \\
\hline Support memory of changes & $\begin{array}{l}\text { Optional scarring to indicate when events have been moved or } \\
\text { deleted }\end{array}$ \\
\hline Error correction & Implement multilevel undo \\
\hline Prioritising intentions & $\begin{array}{l}\text { Implement a means of expressing relative priority of to-do } \\
\text { items (rather than fixed importance levels) }\end{array}$ \\
\hline $\begin{array}{l}\text { Support recording of time-based information } \\
\text { (other than scheduled events) }\end{array}$ & $\begin{array}{l}\text { Implement annotation feature that allows times or time } \\
\text { periods to be annotated. }\end{array}$ \\
\hline $\begin{array}{l}\text { Indicate attributes of events such as } \\
\text { importance, definiteness, movability. }\end{array}$ & $\begin{array}{l}\text { Implement optional marks to assign attributes to events } \\
\text { (note: avoid default values, to avoid unnecessary explicitness) }\end{array}$ \\
\hline Support allocation of preparation time & $\begin{array}{l}\text { Implement a means of adding travelling time to the start of an } \\
\text { event, or more flexible preparation time within a few days } \\
\text { (user-definable) before the event }\end{array}$ \\
\hline \multirow[t]{2}{*}{ Support ambiguity about start and end times } & $\begin{array}{l}\text { Allow start and end times to be allocated a degree of } \\
\text { approximation }\end{array}$ \\
\hline & $\begin{array}{l}\text { Allow events to be linked (e.g. one immediately following } \\
\text { another) }\end{array}$ \\
\hline Event series should match user's understanding & Implement event series as an inheritance hierarchy \\
\hline $\begin{array}{l}\text { Support co-ordinating between user's memory } \\
\text { and diary }\end{array}$ & $\begin{array}{l}\text { Diary needs to be easily accessed and easily viewed, and 'today' } \\
\text { should be easily identifiable }\end{array}$ \\
\hline Support user's memory of making entries & $\begin{array}{l}\text { Entries should be marked (in a hidden but accessible way) } \\
\text { according to who made the entry and when }\end{array}$ \\
\hline \multirow[t]{2}{*}{ Knowing where someone is at the moment } & $\begin{array}{l}\text { Distinguish between events (for that individual) and other } \\
\text { time-based information. }\end{array}$ \\
\hline & Prompt users for location \\
\hline Avoid inappropriate precision & Implement sketching? \\
\hline Support information sharing across tools & Import and export of information between tools \\
\hline $\begin{array}{l}\text { Premature commitment or avoidable } \\
\text { explicitness }\end{array}$ & $\begin{array}{l}\text { Minimise initial demands; allow later addition of information; } \\
\text { minimise defaults or assumptions }\end{array}$ \\
\hline
\end{tabular}

Table 2: Requirements identified in this study

\section{Discussion \& CONCLUSIONS}

Although the diaries used by most respondents in this study conformed to the requirements as identified by Kincaid et al [1], this study identified many further requirements - another layer that was not apparent before such tools had been used in earnest. These requirements are summarised in Table 2. However, the primary finding from this study is that there is no perfect time management tool; an important reason for this is that the same information is used in multiple ways 
for a range of tasks. The information that was originally entered as a personal memory aid may be accessed by others to locate the user, or to identify suitable time for a meeting then, when the time has passed, the same entry may serve as a record of past activity. Some entries involve intention formation [3], while others serve more to set context or provide background information to inform time management. We now consider the issues raised by this study under three headings: the nature of the time management task; the properties of media and tools; and the issue of control and ownership.

\subsection{The nature of the time management task}

Time management combines several kinds of tasks; most existing studies have focused attention on scheduling, group co-ordination and prospective remembering. In particular, Payne [3] addresses the tasks of intention formation and prospective remembering, and Palen [2] identifies six activities that she terms "calendar work":

- Temporal orientation: orienting to events (typically in the future) relative to the present.

- Scheduling: described as a satisficing task of balancing constraints and priorities.

- Tracking: recording information as an event happens, for future reference.

- Reminding: helping users remember to do both scheduled events and associated activities (which may not be recorded).

- Note recording and archiving: to record notes associated with meetings.

- Retrieval and recall: of information previously recorded in the diary.

While all of these tasks are important, they are not the whole story. Time management is a fluid and ongoing activity that incorporates elements of scheduling with elements of reacting to external events and elements of prioritising and restructuring. Current computerised time management tools force all of these tasks into a single mould: that of scheduling.

One of the things that came through strongly in this study, particularly for lecturing staff, is that there is a permanent tension between the demands of meetings, lectures and other items (such as queues of students outside the door) that cannot be put off until later, and the demands of other activities such as research. This can be viewed as a tension between urgency and importance. Of course, this tension has been widely recognised, but shared diaries highlight it. Some respondents stated this defensively; for example, one commented that "Just because I don't have an entry doesn't mean I'm doing nothing", and another said "If I'm not in a meeting, people think it's OK to book my time". Shared diaries demand a high measure of trust, mutual respect, and understanding of the benefits.

The role of visual structure in supporting particular tasks has been discussed above. Mackinlay, Robertson \& DeLine [16] address the challenge of information visualisation in their designs for two diary systems: an individual diary called the Spiral Calendar and a groupware scheduling tool called Time Lattice. Although the focus of their work is on the technology of information visualisation, they do conduct a usability evaluation of the Spiral Calendar, comparing it to a commercially available diary. Both an empirical study and a GOMS-like analysis (reported in more detail by Card 
et al [17]) were conducted, but both focused on pre-defined (well specified) tasks. In particular, they report the time required to locate a target within the diary, as a function of its distance from a starting location. While efficiency on device tasks such as target seeking is one important design consideration, it has little bearing on the overall usability and usefulness of a diary in context.

\subsection{The properties of media and tools}

In this study, several people expressed a desire for a combination of hardware and software systems that would permit them to keep their diary up-to-date and refer to it from all locations (i.e. be portable). Other requirements like visibility and quick accessibility were also mentioned.

In other work settings - notably air traffic control - important properties of different media have been identified. In particular the role of the paper flight strip as both an individual memory aid and also a focal point for co-operative work has been widely recognised. Paper has important affordances and a display structure that has not been replicated by electronic tools. While in air traffic control there are persistent attempts at total computerisation, in order to increase the capacity of the airspace, it would appear that in the case of time management tools the challenge is not to create the perfect tool, but to work towards better integration of tools, exploiting the strengths of all.

It is also clear that there is no universal ideal solution that will satisfy all users, and that different solutions will be optimum for different users. For example, some people intersperse to-dos with other notes in work notebooks while others create master lists and transfer items from one list to another, or manage them using a PDA. In particular, as well as people having personal preferences for the time management strategies and tools they employ, individuals' needs evolve over time (e.g. as their role in the organisation changes, or with the seasons of the academic year), so that the tools that were most appropriate last year may be different from those used currently, and may well be different again in the future. Time management tools need to be flexible and adaptable enough to respond to these changing needs.

On particular feature that has traditionally been recognised as a strength of paper is the support for sketching. Computer based tools to support this are now being developed. For example, Kullberg [6] tests an approach to integrating sketching and speech in the interface of a diary, to allow the user to update the diary contents in a more natural way. However, while he cites the fact that many users mark their diaries using their 'own personal symbolic notation' as a reason for accommodating sketching, in practice the sketch is implemented as an intermediate representation that is interpreted as action, and then fades away. Sketching is exploited more effectively in Dynomite [18], which incorporates selective audio recording with pen-based 'digital ink' while also allowing users to retrospectively code and organise entries. This may bridge some of the existing gulfs between paper-based and electronic tools, exploiting some of the strengths of each.

Sketching's virtue is lack of precision. Inappropriate precision encourages users to trust the information presented; the dangers of this are well illustrated in the accounts by some users of 'critical incidents' where meetings default to inappropriate durations (such as exactly one hour). The dangers of defaults apply both to standard electronic diaries and to systems such as that described by Mueller [7], who describes the implementation of a 'common sense' real world knowledge base to help users manage their time. One example of common sense reasoning 
discussed is the provision of 'intelligent defaults' - e.g. that a meal typically lasts two hours, so a diary booking of two hours will be made as a default for any meal appointment.

This is an example of transfer of control of time management from the user to another agent; the importance of ownership and control emerged in the study particularly with the shift from individual to shared diaries (which affected both manager - PA relationships and also other individuals' sense of control over their time).

\subsection{Making information explicit}

Ultimately, what people do is determined by what they remember. As noted by Payne [3], the act of transcribing helps people to remember, as does asking someone else to remind one. In addition, a variety of external cues are used to support remembering. This is often invaluable; for example, one respondent commented that "Having a list switches off the worry about forgetting". However, diaries, to-do lists and other objects used as reminders can only ever serve as external cues. For managers and PAs, whose time is highly structured by meetings, the external cues are typically the most important, so there is a clear benefit from using shared diaries that easily outweighs the costs; for other staff, the benefits of sharing are not proven. They maintain a greater sense of control over their time management by revealing information selectively, and by making decisions just-in-time. Shared diaries force both explicitness and premature commitment.

Dix et al [19] treat diary systems as just one type of artefact in an organisational memory. They distinguish between triggers (reminders that initiate activity) and placeholders (containers of information), and show how flexibility has to be sacrificed to obtain control. The results from our case study can be interpreted as showing similar conceptions of the role of shared diary systems in an organisation. However, the range of tasks a particular tool is expected to support increases with the shift from individual to shared technologies.

The tasks for shared use require provision of information on: when people are free (e.g. for a meeting); details of meetings; and where people are. These group requirements can often, but not necessarily, be inferred from people's diary entries. In practice, the requirements on shared diaries for supporting group use are different from the requirements on diaries for supporting individuals' time management, for which all kinds of idiosyncratic codes and cross references can be used. It may be that future shared diaries will co-ordinate with personal technologies by filtering information in an appropriate way from the one to the other, but it is clear that the 'one size fits all' approach has fundamental limitations that cannot be addressed by improved design of a single artefact.

\subsection{Implications}

Some previous studies have focused on "barriers" to acceptance of group diaries. Our experience suggests that speaking in terms of barriers (with the implicit assumption that groupware tools are a Good Thing) will limit the outlook of researchers, causing them to miss the essential point that people use a battery of tools, for good reason, to help them manage their time.

Viewed in this way we can see the importance of designing or choosing tools that will fit into a community of other tools. New tools are hardly likely to displace all other resources; instead they 
must find their own niche. To its designers or its purchasers a groupware system may be a tool that will revolutionise future practice in the community, but to its intended users it is just a resource like any other, good for some things and not for others. To evaluate it we must consider not only how well it might fit its intended purpose but also what the competition offers. Perhaps there are other existing resources the fit the intended purpose tolerably well - maybe not as well as the proposed new tool, but well enough; if these pre-existing resources also offer a much better fit for other purposes that are significant to the intended users, then it is quite likely that people will prefer to continue in their present practices.

Mackinlay et al [16] claim that "electronic calendars are gradually becoming more desirable than paper calendars"; the study reported here refutes this claim, showing, rather, that in practice different artefacts are suited to different purposes. No single tool can adequately support all aspects of a professional's time management, and there will always be a tension between the requirement on people to make aspects of their diaries publicly available, to support group working, and individuals' need to have tools that serve the primary function of supporting their memory at minimum possible cost of time.

The issue is not solely one of privacy versus group co-ordination. It is also one of explicitness versus sketchiness. The shift from personal to shared diaries forces people to form intentions earlier than they would naturally choose, so that time management becomes a more explicit and demanding activity. Similarly, the visual structure of scheduling systems forces a variety of activities into a single mould, explicitly either an appointment or a to-do entry. Real users, rather than the simplified users in the designer's mind's eye, use their time management systems as information resources for many kinds of information to be used for many purposes.

Our conclusion, therefore, is that progress in time management (indeed, probably in all types of everyday information resources) should look away from rigidly-defined purposes, explicit precision, and individual tools, and towards fluid usage, vague and provisional information, and harmonious commingling between tools.

\section{ACKNOWLEDGEMENTS}

We are grateful to all the interviewees in this study who gave their time to answering a rather long barrage of questions, and to Harold Thimbleby and anonymous reviewers for constructive criticism of earlier versions of this paper.

\section{REFERENCES}

[1] Kincaid, C. M., Dupont, P. B. \& Kaye, A. R. (1985) Electronic calendars in the office: an assessment of user needs and current technology. ACM Transactions on Office Information Systems. 3.1. 89-102.

[2] Palen, L. (1999) Social, Individual and technological issues for groupware calendar systems. Proc. CHI'99 17-24. New York: ACM Press. 
[3] Payne, S. J. (1993) Understanding calendar use. Human-Computer Interaction. 8. 83-100.

[4] Kovalainen, M., Robinson, M. \& Auramäki, E. (1998) Diaries at work. Proc. CSCW'98. pp. 49-58. New York: ACM Press.

[5] Jackson, M. (1995) Software Requirements and Specifications. Harlow: Addison-Wesley.

[6] Kullberg, R. L. (1995) Mark your calendar! Learning personalised annotation from integrated sketch and speech. CHI Companion'95. 302-303. New York: ACM Press.

[7] Mueller, E. T. (2000) A calendar with common sense. Proc. IUI'2000. 198-201. New York: ACM Press.

[8] Kelley, J. F. \& Chapanis, A. (1982) How professional persons keep their calendars: implications for computerisation. Journal of Occupational Psychology. 55. 241-256.

[9] Beard, D. \& Palanlappap, M. with Humm, A., Banks, D., Nair, A. \& Shan, Y-P. (1990) A visual calendar for scheduling group meetings. Proc. CSCW'90. 279-290. New York: ACM Press.

[10] Grudin, J. and Palen, L. (1995). Why groupware succeeds: discretion or mandate? Proc. European Conf. on Computer-Support of Collaborative Work CSCW'95, 263-278. Dordrecht, The Netherlands: Kluwer.

[11] Brown, B. and Crawshaw, T. (1998) Electronic diaries: how they are used, advice for implementation and a review of products. JTAP report No. 20, available from http://www.jtap.ac.uk/reports/index.htm .

[12] Palen, L. (1998) Calendars on the New Frontier: Challenges of Groupware Technology. Doctoral Dissertation. University of California, Irvine.

[13] Mackay, W. (1990) Users and Customisable Software: A Co-adaptive Phenomenon. Doctoral Dissertation. MIT, Cambridge MA.

[14] Green, T R G (1990) The cognitive dimension of viscosity: a sticky problem for HCI. In D. Diaper, D. Gilmore, G. Cockton and B. Shackel (Eds.) Human-Computer Interaction INTERACT '90. pp 79-86 Amsterdam: Elsevier.

[14] Jones, S. R. \& Thomas, P. J. (1997) Empirical assessment of individuals' 'personal information management systems'. Behaviour and Information Technology. 16.3, 158-160.

[15] Cooper, A. (1999) The Inmates are Running the Asylum. Indianapolis: Sams Publishing.

[16] Mackinlay, J. D., Robertson, G. G. \& DeLine, R. (1994) Developing calendar visualizers for the information visualizer. Proc. UIST'94. 109-118. New York: ACM Press.

[17] Card, S. K., Pirolli, P. and Mackinlay, J. D. (1994) The cost-of-knowledge characteristic function: display evaluation for direct-walk dynamic information visualizations. In Adelson, B., 
Dumais, S. and Olson, J. (Eds.) CHI '94: Human Factors in Computing Systems. New York: ACM Press.

[18] Wilcox, L. D., Schilit, B. N. \& Sawhney, N. (1997) Dynomite: a dynamically organised ink and audio notebook. Proc. CHI'97. 186-193. New York: ACM Press.

[19] Dix, A., Wilkinson, J. \& Ramduny, D. (1998) Redefining organisational memory — artefacts, and the distribution and co-ordination of work. In Proc. Workshop on Understanding Work and Designing Artefacts. University of York. pp.39-49. 


\section{APPENDIX: THE QUESTIONNAIRE}

\section{Diaries questions:}

1. How many diaries do you keep?

2. What kind(s) of diaries do you use?

3. Where do you keep your diary(s)?

4. How important is a diary to your work?

5. Does anyone else read your diary?

6. Does this affect what you write in any way?

7. Does anyone else write in your diary?

8. How are you notified of any changes, such as additions, deletions or re-orderings?

9. How many of your appointments get noted?

10. From what sources do appointments typically arise?

11. Do you use your diary to record other information?

12. How do you deal with regular or routine appointments?

13. (If electronic): Do you annotate your appointments in any way (e.g. colour)?

14. Do you have a regular system for annotation, or is it just ad hoc?

15. [If regular] Is it personal or do other people use it?

16. Does it work?

17. What is it?

18. Do you ever change the system?

19. How do you deal with changes to, or cancellations of, appointments?

20. What is your pattern for reading your diary?

21. (If multiple) How do you co-ordinate your diaries?

22. What happens when your diary is inaccessible? (Computer broken, or you away from the office, etc)

23. Can you recall any critical incidents in which your diary keeping failed?

24. Is there anything else about your time management that we haven't covered?

\section{To-do lists:}

25. Do you make to-do lists?

26. What form are your to-do lists (e.g. paper / electronic)?

27. How important are they to your work (or home life)?

28. How many do you make, and how regularly do you make them?

29. Where do you make them?

30. What time-window do they cover?

31. Do you edit your to-do lists?

32. Do you ever copy items from one list to another?

33. Do you have a routine pattern for consulting to-do lists?

34. Do you recall any critical incidents involving to-do lists?

35. Is there anything else about such lists that we haven't covered?

\section{Related issues:}

36. Do you intentionally use any other mechanisms to help you manage your time or remember about things you intend to do?

37. Do you ever consult colleagues' electronic diaries?

38. If so, how frequently, and for what purposes?

39. Do you use the multi-user features of meeting-maker to set up meetings or to-do lists?

40. If so, can you give some typical scenarios of the way you use it?

41. Can you recall any critical incidents relating to multi-user diaries?

42. Do you often have multiple to-dos or appointments that depend on each other?

43. If so, how do you keep track of the dependency?

44. What, to you, are the advantages of paper, individual PC, shared PC, lap-top, palm-top, \& wristwatch systems? 